\title{
The Use of MCS-51 Parallel Port to Simulate Digital Potentiometer
}

\author{
Jinlan Zhang, Mei Lin, Zeli Zhang \\ Dept. Electrical Engineering, Binzhou Vocational College, Binzhou, China \\ zj17298@163.com, zh5608@163.com
}

Keywords: DCP; power resistance; $51 \mathrm{MCU}$; P0 port

\begin{abstract}
Digital potentiometer is another form of DAC circuit, which has been widely applied in the measurement and control(MAC). But, the commonly used of digital potentiometer resistance value is limited to $10 \mathrm{k} \Omega, 50 \mathrm{k} \Omega, 100 \mathrm{k} \Omega, 200 \mathrm{k} \Omega$, and its change of resistance is linear. This is often not suitable for the needs of the measurement and control. And digital potentiometer chip is imported products, not easily to buy and it is with higher cost. $51 \mathrm{MCU}$ is cheap to buy, there is not pull-up resistors in its P0 port, and by connecting power resistor can simulate simple digital potentiometer. If other parallel port is used, cooperate with analog switch CC4066 can simulate digital potentiometer, and the resistance can choose according to the need.
\end{abstract}

\section{Introduction}

Digital potentiometer is called numerical control potentiometer (digitally controlled potentiometers, DCP), can replace the traditional mechanical potentiometer, overcome the prone to bad contacts of mechanical potentiometer, generate electrical noise and not easy to achieve automatic control of faults, easy to realize automatic control.

Digital potentiometer are analog-digital mixed signal product, is a kind of step adjustable resistor. Its input signal is digital, output signal is analog, it's a special kind of D/A converters. But its output is not a voltage or current, but the ratio of resistance or resistance, therefore, also known as resistive digital-to-analog converters (RDAC).Most common digital potentiometer using SPI bus or I2C bus to communicate with the computer.

The commonly used digital potentiometer resistance is linear change. There are three outputs, respectively is $\mathrm{L}, \mathrm{H}, \mathrm{W}$. The equivalent circuit is shown in figure 1.

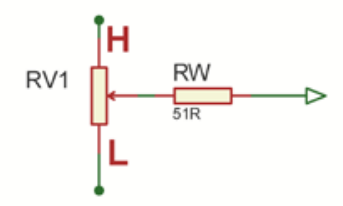

Figure 1. Digital Potentiometer Equivalent Circuit

The common digital potentiometer is in order to 7 bit or 8 bit in the majority, There are four resistance value of $\mathrm{R}_{\mathrm{HL}}: 10 \mathrm{k}, 50 \mathrm{k}, 100 \mathrm{k}, 200 \mathrm{k}$.

Digital potentiometer resistance has a $10 \mathrm{k}, 50 \mathrm{k}, 100 \mathrm{k}, 200 \mathrm{k}$, the limitations of value choice. Resistance change is linear, this is often difficult to meet the requirements of control. Digital potentiometer is not very easy to get device, there is no domestic production of products, to buy more expensive imported products.

If the use of 51 MCU P0 port no pull-up resistance characteristics, can also be used by its P0 port do analog switch, simulating the potentiometer, this value can be selected according to need. If have analog switch CC4066, also can adopt the method of resistors in series to realize the function of numerical control potentiometer.

\section{Shunt resistance method to realize simple DCP}

Shunt resistance method does not need to increase the peripheral chips, but the output voltage 
level is not much, only applies to places with low output voltage change requirements.

\subsection{Working principle of the circui}

As shown in figure 2, 8 switches can be combined into 256.

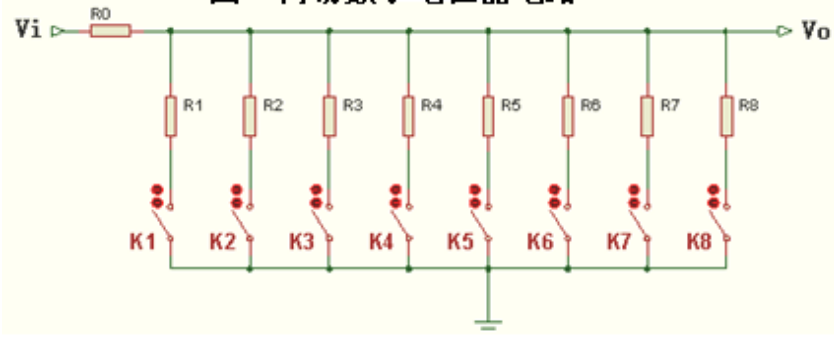

Figure 2. Simple Digital Potentiometer Circuit

The output voltage $. \mathrm{V}_{0}=\frac{1}{\mathrm{R}_{0} \sum_{1}^{8} \frac{1}{\mathrm{R}_{\mathrm{n}}}+1} \mathrm{~V}_{\mathrm{i}}$

Type in Rn as either numerical resistor form R1 to R8.

Through the control of switch K1 K8, so the control system for the output voltage.51 MCU P0 mouth supreme pull resistance, eight switch can replace in figure 2, realize simple analog output, as shown in figure 3. When a certain output of P0 mouth low electricity at ordinary times, equivalent to the bit corresponding to the switch, when one output high electricity at ordinary times, P0 mouth is equivalent to the level switch.

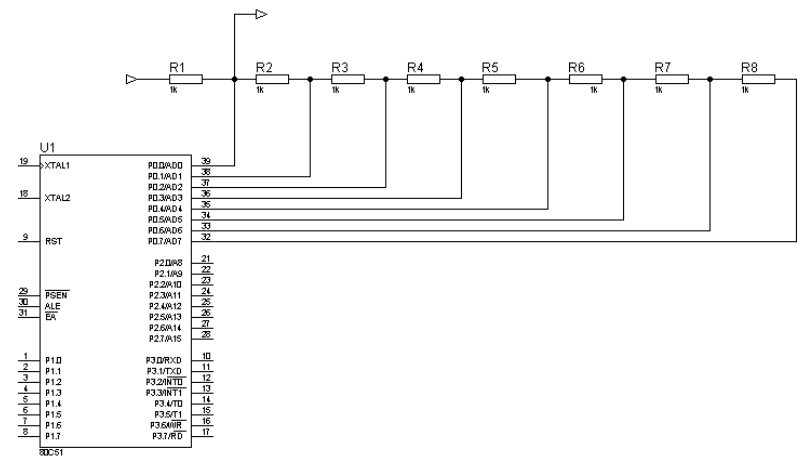

Figure 3. MCU Control Analog to Digital Potentiometer

$\mathrm{R} 1$ to $\mathrm{R} 8$ values, in line with the weight ratio of relations, namely, R2=2R1, R3=4R1, R4=8R1, $\mathrm{R} 5=16 \mathrm{R} 1, \ldots ., \mathrm{R} 8=128 \mathrm{R} 1$. All the switch is closed, that is, $\mathrm{P} 0=0 \mathrm{x} 00$, all resistance in parallel is about R1, all switch off are $256 \mathrm{R} 1$.

P0 output corresponding data, can achieve equivalent resistance in the order: $\quad \frac{1}{2} \frac{1}{2} \quad \mathrm{R} 1, \quad \frac{2}{3} \quad \mathrm{R} 1, \mathrm{R} 1, \quad \frac{4}{3} \quad \mathrm{R} 1,2 \mathrm{R} 1, \quad \frac{8}{3} \quad \mathrm{R} 1,4 \mathrm{R} 1, \quad \frac{16}{3} \quad \mathrm{R} 1,8 \mathrm{R} 1, \quad \frac{32}{3} \quad \mathrm{R} 1,16 \mathrm{R} 1 \quad$ 、 $\frac{64}{3} \quad \mathrm{R} 1,32 \mathrm{R} 1, \quad \frac{128}{3} \quad \mathrm{R} 1,64 \mathrm{R} 1, \quad \frac{256}{3} \quad \mathrm{R} 1,128 \mathrm{R} 1, \quad \frac{516}{3} \quad$ R1.Among them $\frac{1}{2} \mathrm{R} 1,1 \mathrm{R} 1,2 \mathrm{R} 1,4 \mathrm{R} 1,8 \mathrm{R} 1,16 \mathrm{R} 1,32 \mathrm{R} 1,64 \mathrm{R} 1,128 \mathrm{R} 1$, Corresponding with the digital output 1,2,4,8,16,32,64,128,256, With high precision . Among them $\mathrm{R} 1, \frac{16}{3} \mathrm{R} 1, \frac{32}{3} \mathrm{R} 1, \frac{64}{3} \mathrm{R} 1, \frac{128}{3} \mathrm{R} 1, \frac{256}{3} \mathrm{R} 1, \frac{516}{3} \mathrm{R} 1$ Corresponding 3, 5, 11, 21, 43, 85, 172, low precision, and there is no intermediate value, so that we can get close to linear change value.

So if converted to 8 bits of data, conversion precision mainly depends on resistor R1 R9 resistance, if choose precise resistance, will have higher accuracy. If converted into eight or more data, not only has discrete, and error in calculation, so precision is low. The combination of switch state has 256 kinds, but this way only use part of the configuration. 
Table 1 . P0 port output data and the relationship between the equivalent resistance

\begin{tabular}{|l|l|l|l|l|l|l|l|l|l|}
\hline Output & 0.5R1 & 1R1 & 2R1 & 4R1 & 8R1 & 16R1 & 32R1 & 64R1 & 128R1 \\
\hline P0 & 0x00 & 0xfe & 0xfd & 0xfb & 0xf7 & 0xef & 0xdf & 0xbf & 0x7f \\
\hline Output & 0.67R1 & 1.33R1 & 2.67R1 & 5.33R1 & 9.67R1 & 21.33R1 & & 256R1 & \\
\hline P0 & 0xfc & 0xfa & 0xe7 & 0xcf & 0x9f & 0x3f & & 0xff & \\
\hline
\end{tabular}

Table 2 Output voltage and the relationship between data output port P0

\begin{tabular}{|l|l|l|l|l|l|l|l|l|l|}
\hline P0 & 0x00 & 0xfe & 0xfd & 0xfb & 0xf7 & 0xef & 0xdf & 0xbf & 0x7f \\
\hline $\mathrm{V}_{\mathrm{O}}$ & $1.4 \mathrm{~V}$ & $1.485 \mathrm{~V}$ & $1.62 \mathrm{~V}$ & $1.89 \mathrm{~V}$ & $2.43 \mathrm{~V}$ & $3.51 \mathrm{~V}$ & $5.67 \mathrm{~V}$ & $9.99 \mathrm{~V}$ & 18.36 \\
\hline $\mathrm{P} 0$ & $0 x f \mathrm{C}$ & $0 x f 9$ & $0 x f 3$ & $0 x e 7$ & $0 x c f$ & $0 x 9 f$ & $0 x 3 f$ & & \\
\hline $\mathrm{V}_{\mathrm{O}}$ & $1.44 \mathrm{~V}$ & $1.57 \mathrm{~V}$ & $1.71 \mathrm{~V}$ & $2.06 \mathrm{~V}$ & $2.79 \mathrm{~V}$ & $4.23 \mathrm{~V}$ & $7.11 \mathrm{~V}$ & & \\
\hline
\end{tabular}

In most cases, the need is the output resistance tolerance of the control, the parallel resistance value can be selected according to need. When you don't have to consider the impact of input control data and output resistance, use an array to determine the input data in the programming and the relationship between the output resistance, resistance to eight different parallel combination has 256 kinds of state. If resistance still according to the above relations, P0 mouth corresponding output low electricity at ordinary times, appropriate grounding resistance, so the relationship between resistance and P0 output, and can be used: Where $\mathrm{n}$ take $1 \sim 8$, the corresponding resistor serial number, $\mathrm{B}_{\mathrm{n}-1}$ is corresponding to a value. $\mathrm{P} 0=0 \mathrm{xf} 5$ when, for example, only grounding resistor $\mathrm{R} 1, \mathrm{R} 3$, the resistance to ground.

If directly with the method of look-up table when programming, if array length is 256 elements, can cause compilation errors. We can separate the two and a half a byte, composed of two 16 elements of an array, can solve this problem.

unsigned char table 1[]$=\{0 \times F 0,0 \times 70,0 \times B 0,0 \times 30,0 \times D 0,0 \times 50,0 \times 90,0 \times 10,0 \times E 0$,

0x60,0xA0,0x20,0xC0,0x40,0x80,0x00\};

unsigned char table2[] $=\{0 \mathrm{x} 0 \mathrm{f}, 0 \mathrm{x} 07,0 \mathrm{x} 0 \mathrm{~b}, 0 \mathrm{x} 03,0 \mathrm{x} 0 \mathrm{~d}, 0 \mathrm{x} 05,0 \mathrm{x} 09,0 \mathrm{x} 01,0 \mathrm{x} 0 \mathrm{e}$,

0x06,0x0a,0x02,0x0c,0x04,0x08,0x00\};

To get the value of resistance tolerance of the sample, we can make the $\mathrm{P} 0=$ table $[\mathrm{x}] 1 \mid$ table2 [y].

\subsection{Examples of application}

Application of simulate to digital potentiometer made simple numerical control voltage source, the circuit principle as shown in figure 4.

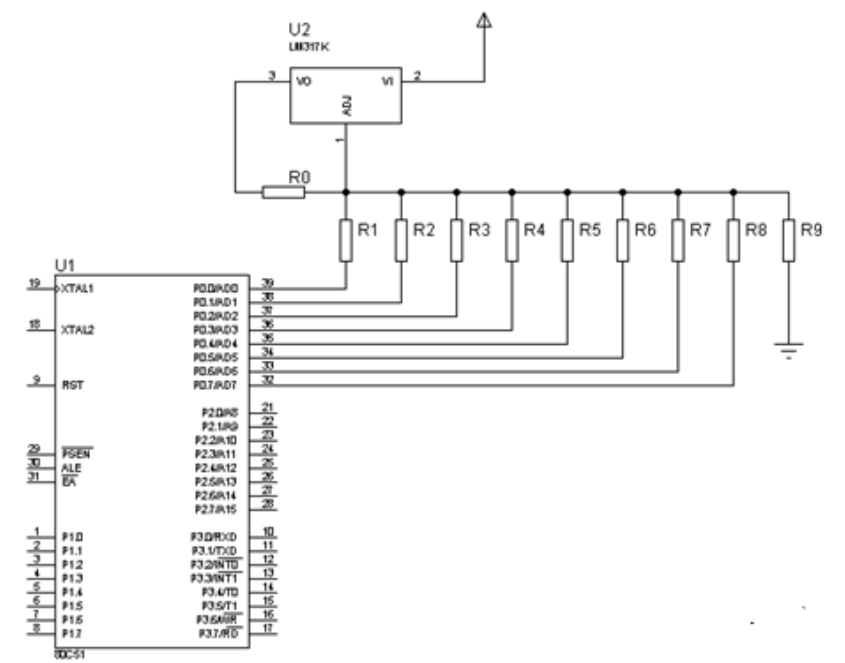

Figure 4. Analog to Digital Dotentiometer Constitute the Vumerical Control Voltage Source

CW317 is three-terminal can assemble into voltage regulator, the maximum input voltage 30v, output voltage can be adjusted between $1.3 \sim 30 \mathrm{v}$. In fact use CW7805 also can realize the output 
voltage is adjustable, the maximum input voltage 30v, but between $5 \sim 30 \mathrm{v}$ output voltage regulation.

Shown in figure 4 adjustable voltage source of 51 single-chip microcomputer control circuit, can by the single chip microcomputer control the output voltage, power supply voltage $14 \mathrm{v}$ (car generator output voltage), through the adjustable three-terminal voltage regulator output dc voltage is $1.4 \sim 12.4$.Can be used for lighting levels or pulse drive the speaker volume control. Take $1 \mathrm{k}$ if R0, take 100R1, R2 R8 value $200 \sim 12800$ ohms.

Output voltage calculation:

Table 2 output voltage relationship with P0 mouth output data

If the power supply voltage of $14 \mathrm{~V}$, the output voltage of $12.4 \mathrm{~V}$, the biggest such gain 17 different voltage output. It meet the requirements of the output voltage change is not used in high places.

Write the driver can use look-up table method.

\section{C4066 analog switch resistance in series method}

Analog switch is a common CMOS digital integrated circuit, cheap tesco. Use analog switch and resistor in parallel, can simulate digital potentiometer, the resistance can be linear change, can also according to the index law or logarithmic law changes.

\subsection{Simulate the 4-bit digital potentiometer}

The circuit shown in figure 5 needs one CC4066, four high precision of resistance and one 51 MCU, resistance changes by analog switch control. When P1 port output “OXXF”, all switches are connected, the output value is "0"(Ignore the pressure drop of analog switch). When P1 port output " $0 \mathrm{xX0}$ ", all switches are disconnected, the output value is 4 series resistance value. Four is proposed. Theoretical meet R0 $=8 \mathrm{R} 3, \mathrm{R} 1, \mathrm{R} 2, \mathrm{R} 3=4=2 \mathrm{R} 3$, R3 relations. So, resistance is proportional to the right of a resistance value, thus can be called power resistance. And so , output resistance value between $0 \sim 15 \mathrm{r} 3$ changes, the equivalent of four conversion accuracy.

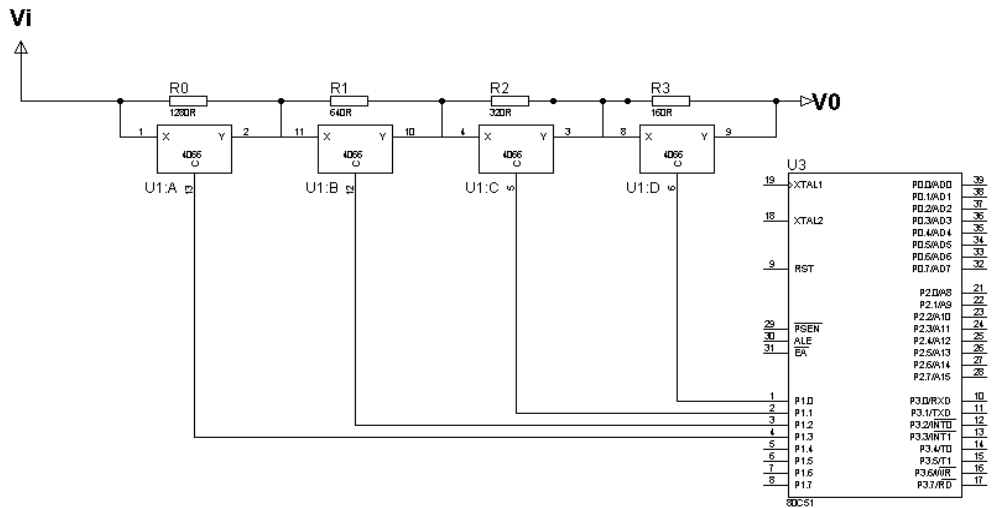

Figure 5.Four Series Analog Potentiometer

It is clear that there are good linear relationship in the circuit, only have less digits. Resistance tolerance relation with $\mathrm{P} 0$ output parameters, $\mathrm{R}_{\mathrm{z}}=-\mathrm{P}_{0} \mathrm{R}_{3}$.If need to change resistance according to the index law, only need to change the value corresponding to the resistance in the table.

Table 3. Linear analog potentiometer resistance selection

\begin{tabular}{|l|l|l|l|l|l|l|l|l|l|}
\hline P0 & 0x00 & 0xfe & 0xfd & 0xfb & 0xf7 & 0xef & 0xdf & 0xbf & 0x7f \\
\hline $\mathrm{V}_{\mathrm{O}}$ & $1.4 \mathrm{~V}$ & $1.485 \mathrm{~V}$ & $1.62 \mathrm{~V}$ & $1.89 \mathrm{~V}$ & $2.43 \mathrm{~V}$ & $3.51 \mathrm{~V}$ & $5.67 \mathrm{~V}$ & $9.99 \mathrm{~V}$ & 18.36 \\
\hline P0 & $0 x f \mathrm{c}$ & $0 x f 9$ & $0 x f 3$ & $0 x e 7$ & 0xcf & 0x9f & 0x3f & & \\
\hline $\mathrm{V}_{\mathrm{O}}$ & $1.44 \mathrm{~V}$ & $1.57 \mathrm{~V}$ & $1.71 \mathrm{~V}$ & $2.06 \mathrm{~V}$ & $2.79 \mathrm{~V}$ & $4.23 \mathrm{~V}$ & $7.11 \mathrm{~V}$ & & \\
\hline
\end{tabular}

\subsection{Simulate the 8-bit digital potentiometer}

If we use two pieces of CC4066, cooperate with 8 high precision resistor, precision can achieve 256 digital potentiometer. Resistance values for R7 reference, R6, R5, R4, R3, R2, R1, R0 R7 of 2, 4, 
$8,16,32,64,128$ times.P0 port output meet $R_{z}=P_{0} R_{3}$, just $P 0$ values of 8 bit binary number. As shown in figure 6 is 8 bit series analog digital potentiometer.

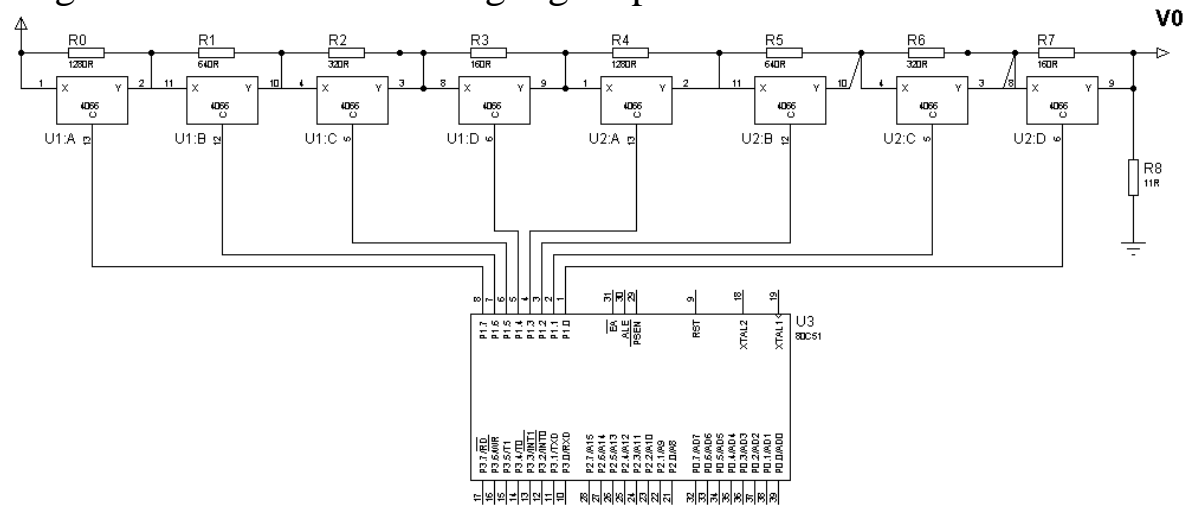

Figure 6 .Simulate Digital Potentiometer Composed of Two Pieces of CC4066

\subsection{Use method of weighted-resistor in series to Realize DAC function}

VREF is reference voltage, when $P 0=0 x f f$, the output voltage $V_{O}=V_{R E F}$.

When $\mathrm{P} 0=0 \mathrm{x} 00$, all analog switch are disconnected, the total value of series resistance is 255 times of R8, the output voltage $\mathrm{V}_{\mathrm{O}}=\mathrm{V}_{\mathrm{REF}} / 256$.

When $\mathrm{P} 0=0 \mathrm{x} 01$, only P1.0 port control of analog switch and other switch, R7 resistance equals the R8, due to the resistance was short circuit, so the total resistance in series is R8 of 254 times, so the output voltage $\mathrm{V}_{\mathrm{O}}=\mathrm{V}_{\mathrm{REF}} / 255$.

When $\mathrm{P} 0=0 \mathrm{xff}$, all of the switch connect, At this time, the output voltage $V_{O}=V_{R E F}$.

The output voltage : $V_{o}=\frac{R_{8}}{R_{8}+\sum_{0}^{7}\left(B_{n} R_{n}\right)} V_{R E F}$

Problem: when P0 output is 0 , the output voltage is not zero, only about $1 / 256$ of the reference voltage. From the expression, the output voltage and input data is an approximate linear relationship.

If we remove R0, such when P1.7 port output low level, the corresponding analog switch is disconnect, equivalent series resistance is close to infinity, realize the output is 0 .This bit can only make a separate control bits, only when $\mathrm{P} 0=0 \mathrm{x} 00$ it can be disconnected, other condition through. When P0 $=0$ x80, output voltage $V_{O}=V_{R E F} / 128$.In this way, we can obtain good linear effect, but need to simply process the data .

The output data 0x00 into 0 x00 output from P1 port, Output data 0x01 into 0x80 output from P1 port. We can use the look-up table method to achieve this transformation.

This DAC circuit, we just need to output data directly to P0 port, therefore it can simplify the process.

\section{Using 16 choose 1 analog switch constitute 4-bit digital potentiometer}

CC4067 is 16 choose 1 analog switch, made simple digital potentiometer CC4067 combining exhaust resistance, occupy less port, combined with the resistance consistency, can obtain higher precision. Circuit shown in figure 7 , the 2 row resistance constitutes the digital potentiometer composed of 16 resistance $(1 \mathrm{k})$. In line with the digital potentiometer device:

$$
R_{H L}=\frac{2^{n}-D}{2^{n}} R_{H L}+R_{W}
$$




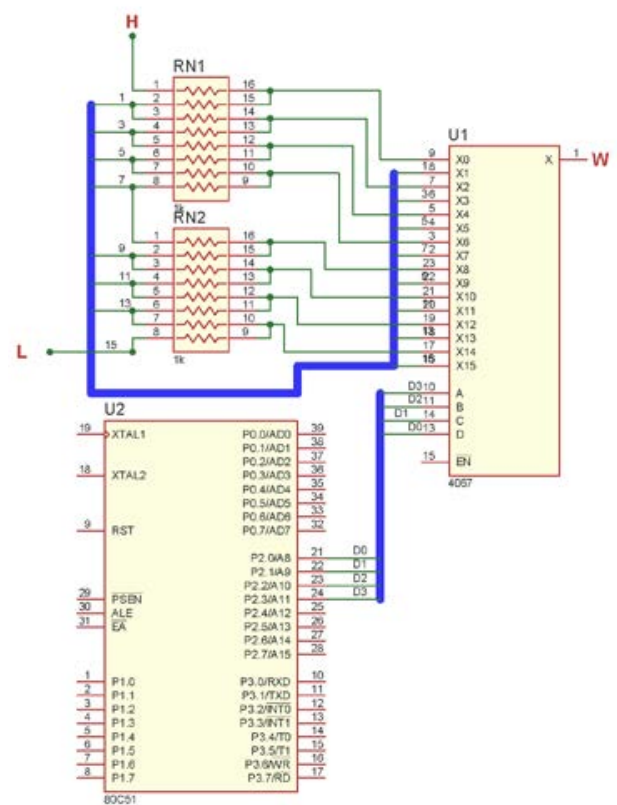

Figure 7. CC4067 Constitute Digital Potentiometer

\section{Conclusion}

Use P0 mouth control resistance grounding way of parallel type analog to digital potentiometer, only need 8 high precision metal film resistors can only, simple circuit, low cost, and can meet the requirements is not high. Such as in car siren control, to control the volume, can completely meet the requirements.

Other port outside of using P0 cooperate CC4066 constitute a series of digital analog switch circuit potential, the disadvantage is that there are multiple analog switch series, analog switch is about $0.1 \mathrm{~V}$ voltage, thus lead to affect the conversion accuracy, can only be used for precision occasions with low requirements. This way can realize linear resistance change, also can realize the nonlinear resistance change, can be used as a digital potentiometer, the output voltage can also be used as a type of DAC, can replace DAC module USES.

USES the multi-channel analog switch and use 16 selected row resistance constitute a digital potentiometer, has high conversion accuracy, but less digits.

The scheme has low cost, short program in, has high reference value.

The above circuit through simulation and actual circuit test, available proved feasible.

\section{References}

[1] Zhanyou Sha, Digital potentiometer application guide ,1st ed. Beijing: Chemical industry press, pp. 2-5, March 2008.

[2] Quanli Li, The principle and application of singlechip microcomputer technology, 2sec.ed. Beijing: Higher education press, pp.20-22, September 2000.

[3] Xicai He, Commonly used integrated circuit applications, 1st ed. Beijing: Electronic industry press, pp.316, June 2007.

[4] Xinxian Wang, General IC quick manual, sec.ed.Jinan: Shandong science and technology press,pp.90,2005.

[5] Yuanyuan Huang,Jianxun Chen, Embedded Automatic Flute Recognition System Based on ARM, Industrial control computer, vol.3,2011. pp. 36-37. 